\title{
Diseño e implementación de un sistema de control para mejorar la calidad de los gases de combustión de una caldera pirotubular de 5 BHP
}

\section{(Design and implementation of a control system to improve the quality of the combustion gases in the fire-tube boiler of 5 BHP)}

\author{
Carlos Alfredo Pérez Albán', Alexis Cordovés García, Jorge Román Terán \\ Benalcázar $^{1}$
}

\begin{abstract}
Resumen:
La presente investigación tiene como objetivo el diseño e implementación de un sistema para el control de la calidad de los gases de combustión de una caldera pirotubular de 5 BHP. En base al porcentaje de $\mathrm{O}_{2}$ presente en los gases de combustión, medido mediante una sonda Lambda, se determina el porcentaje de $\mathrm{CO}_{2}$ que se emite a la atmósfera. Un control proporcional PID se encarga de la regulación automática de la entrada de aire a la caldera mediante un actuador, según el valor de la concentración de oxígeno registrado en los gases de combustión. El sistema de control cuenta con una pantalla HMI y un PLC modular. Los resultados alcanzados aseguran la emisión de gases contaminantes dentro de los parámetros establecidos por las normas ambientales vigentes lográndose así, la calidad requerida de los gases de combustión y la reducción del consumo de combustible de la caldera.
\end{abstract}

Palabras clave: caldera de vapor; sonda lambda; pantalla HMI; control lógico programable

\begin{abstract}
:
The goal of this paper is the design and implementation of a system for controlling the quality of the combustion gases in a fire-tube boiler of $5 \mathrm{BHP}$. Based on the percentage of $\mathrm{O}_{2}$ present in the combustion gases, measured by a lambda sensor, the percentage of $\mathrm{CO}_{2}$ emitted into the atmosphere is determined. PID proportional control is responsible for the automatic regulation of the entry of air to the boiler by an actuator, according to the percentage of the oxygen concentration in the combustion gases. The control system has an HMI display and a modular PLC. The results achieved ensure pollutant gases emissions within the parameters established by current environmental standards, achieving the required quality of combustion gases and reducing the fuel consumption of the boiler.
\end{abstract}

Keywords: steam boiler; lambda sensor; HMI display; programmable logic control

\footnotetext{
${ }^{1}$ Universidad Tecnológica Equinoccial, Santo Domingo - Ecuador ( \{paca504082, alexis.cordoves\}@ute.edu.ec; jteran@quemco.com )
} 


\section{Introducción}

Los problemas asociados a la contaminación ambiental, fundamentalmente en las grandes ciudades, continúa siendo un tema de gran preocupación para los gobiernos y organizaciones ambientalistas. Sin embargo, no se cuenta en muchos casos con el soporte tecnológico para dar seguimiento al índice de contaminación provocado por las diferentes fuentes emisoras.

Se entiende por contaminación ambiental: "la presencia en la atmósfera de sustancias producidas por actividades humanas o procesos naturales que causan efectos adversos al hombre y medio ambiente" (Ministerio del Ambiente, 2007, pág. 5). Los contaminantes atmosféricos más frecuentes y ampliamente dispersos son: $\mathrm{CO}, \mathrm{SO}_{2}, \mathrm{NO}_{x}, \mathrm{CO}_{2}$ y el PM2,5.

La Organización Mundial de la Salud sitúa el umbral de salubridad del valor de material particulado PM 2,5 en una media anual de $25\left(\mu \mathrm{g} / \mathrm{m}^{3}\right)$; como se conoce, estas partículas tan pequeñas tienen un efecto perjudicial a la salud y se tornan particularmente peligrosas en ciudades como Pekín que registró en el año 2014 una media de 85,9 $\mu \mathrm{g} / \mathrm{m}^{3}$ según el editorial DiarioLibre.com del 16 de abril del 2015. Linares, C. y Díaz, J. (2015) publicaron el registro de la concentración de las partículas PM 2,5 de un día laboral en la ciudad de Madrid con valores que oscilaron de $68 \mu \mathrm{g} / \mathrm{m}^{3}$ a las 15 horas a $74 \mu \mathrm{g} / \mathrm{m}^{3}$ a las 19 horas. México y Ecuador, en el área latinoamericana, tienen una norma de valor medio anual de $15 \mu \mathrm{g} / \mathrm{m}^{3}$. Sin embargo, hay informes que denotan registros superiores.

Existen tres grandes fuentes de contaminación del aire provenientes de actividades humanas: fuentes estacionarias, móviles y de interiores. Constituyen fuentes estacionarias las industrias y las plantas de producción de energía, son fuentes móviles los vehículos motores y fuentes interiores los hogares o viviendas. A los efectos de la presente investigación son de interés las fuentes estacionarias tipo industrias, específicamente las calderas de vapor. Esta fuente puede provocar afectaciones de manera puntual o a una región mediante varias fuentes pequeñas independientes (Ministerio del Ambiente, 2007).

Las calderas de vapor se clasifican según: la disposición del fluido, su circulación, el mecanismo de transmisión del calor dominante, su estructura, modo de intercambio de calor, entre otros factores. Según la clasificación dada por Alvarez (2006, pág. 6), la caldera objeto de estudio atendiendo a su diseño y capacidad se clasifica como: caldera de generación de vapor y agua sobrecalentada para uso industrial, de combustible líquido, de diseño pirotubular o de tubos de humos de $5 \mathrm{BHp}$.

Los procesos de combustión dan lugar, inevitablemente, a la emisión a la atmósfera de sustancias que modifican la composición y que tienen un poder contaminante sobre el aire, la tierra y el agua. Al asegurar la calidad requerida de la mezcla aire-combustible durante el proceso de combustión, 
se reduce el impacto negativo sobre el medio ambiente de los gases emanados de dicho proceso (Kojan, A. L., 2000).

Existen diferentes métodos de control de la calidad de la combustión. Se diferencian en el tipo de accionamiento de los elementos reguladores de la entrada de aire y de combustible y en la forma de captura de la información sobre la composición de la mezcla. Uno de los métodos más empleados es el de corrección de medida. La corrección se realiza al parámetro de medición elegido $\left(\mathrm{O}_{2}, \mathrm{CO}_{2}, \mathrm{CO}\right)$. Pese a que el control mediante $\mathrm{CO}_{2}$ es más preciso, el costo de importación de los sensores es elevado y no están disponibles comercialmente en el país. Por esta razón, el control mediante la medición de $\mathrm{O}_{2}$ es el más utilizado a nivel industrial en Ecuador.

Las calderas normalmente son instrumentadas para controlar el valor de parámetros como la temperatura y la presión, también puede controlarse la calidad de los gases de combustión. Con este fin, es utilizada una sonda lambda (Sonda- $\lambda$ ) para medir la concentración de oxígeno en los gases de escape antes de que sufran alguna alteración. Se toma como referencia el coeficiente de aire (lambda) con valor 1 cuando la relación aire/combustible es estequiométrica o ideal y corresponde a la relación de mezcla de 14,7/1. Si el valor de lambda es mayor que 1 , se entiende que la mezcla es rica en oxígeno y si es menor que 1 se entiende que la mezcla es pobre en oxígeno. . La medida del oxígeno representa del grado de riqueza de la mezcla, magnitud que la sonda transforma en un valor de tensión y que comunica a la unidad de control (Salinas Villar, 2006).

La utilización de sistemas de Interface Hombre-Máquina (HMI) acoplados con un Control Lógico Programable (PLC) en la industria contemporánea es creciente, estos sistemas desempeñan un papel fundamental en el diseño de una solución de automatización verdaderamente esbelta, logran una adecuada combinación de la visualización y el control del funcionamiento de las máquinas.

La estructura básica de un sistema de control HMI-PLC incluye sensores, actuadores, interfaz de operador y dispositivos de control lógicos. Los sensores miden las variables físicas, como la temperatura, flujo, presión, etc. y convierten esa información en una señal eléctrica, el dispositivo actuador transfiere la información al HMI, y el dispositivo lógico controla el funcionamiento de la máquina. El dispositivo lógico examina las entradas realizadas por el operador y por el sensor, y envía señales al dispositivo actuador. Este modelo se aplica a procesos discretos y continuos.

El objetivo del presente artículo es el diseño e implementación de un sistema de control de combustión con HMI, para asegurar la adecuada relación aire-combustible durante la combustión en una caldera pirotubular de $5 \mathrm{BHP}$, que permita reducir las emisiones de gases contaminantes a la atmósfera y el uso racional de combustible. 


\section{Materiales y Métodos}

En la presente investigación se ha empleado el método de corrección por medida de $\mathrm{O}_{2}$ para controlar la calidad de los gases de combustión y se ha utilizado el diésel como combustible. El sistema propuesto funciona basado en el lazo de control mostrado en la Figura 1 mediante el que se realiza la medición del oxígeno residual en los gases de combustión para determinar si se cumple la estequiometría de la mezcla aire/combustible es decir, la relación de mezcla de 14,7/1. Para ello, se fija un valor de "SET POINT" del porcentaje de oxígeno según el tipo de combustible, que para el diésel el valor recomendado se encuentra entre $\left(3,7-5 \% \mathrm{O}_{2}\right)$. En caso de no cumplirse la relación estequiométrica el controlador de aire modula el ingreso de aire hasta alcanzar el valor "SETEADO" del porcentaje de oxígeno.

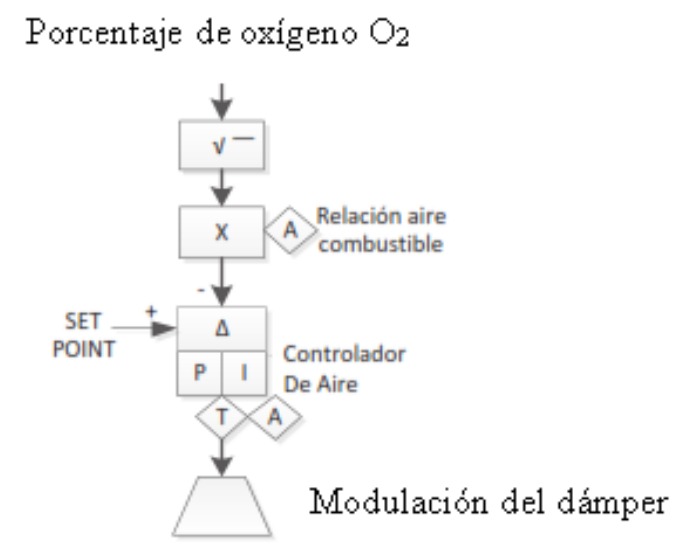

Figura 1: Estructura básica del lazo de control para el sistema propuesto

Para medir la concentración de oxígeno presente en los gases de combustión de la caldera se utilizó una sonda lambda; fue necesario determinar la función que relaciona el voltaje emitido por la sonda a partir el porcentaje $\mathrm{O}_{2}$ presente en dichos gases. Para ello, se provocó la variación del porcentaje de $\mathrm{O}_{2}$ en condiciones de presión y temperatura normales (TPN) en un ambiente cerrado, dicha variación fue registrada mediante un equipo analizador de gases de la marca BrainBee y se tabuló el voltaje medio emitido por la sonda lambda en función del valor de concentración de $\mathrm{O}_{2}$ entregado por el equipo analizador, aplicándose el método de calibración comparativo (Ver Figura 2).

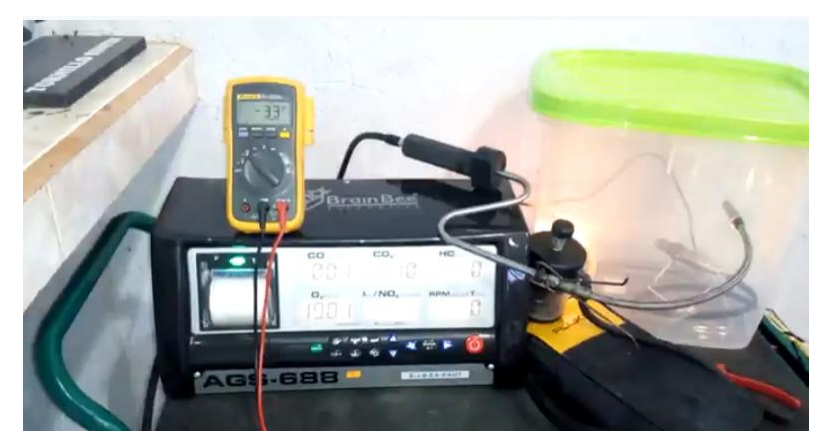

Figura 2: Pruebas de paralelaje entre sonda lambda y analizador de gases Brain Bee. 
Así, se determinó que la sonda lambda emite un valor de $-34 \mathrm{mV}$ a una concentración del $20.8 \%$ de oxígeno, que es la concentración de oxígeno diatómico presente en el volumen de la atmósfera terrestre. Al disminuir paulatinamente la concentración de oxígeno, el voltaje de la sonda lambda se incrementa yendo desde los $-34 \mathrm{mV}$, hasta un valor de $100 \mathrm{mV}$ que equivale a una concentración de $2 \%$ de oxígeno. En la Figura 3 puede observarse que los valores obtenidos de voltaje de la sonda contra concentración de oxígeno se corresponden con la ecuación de una recta.

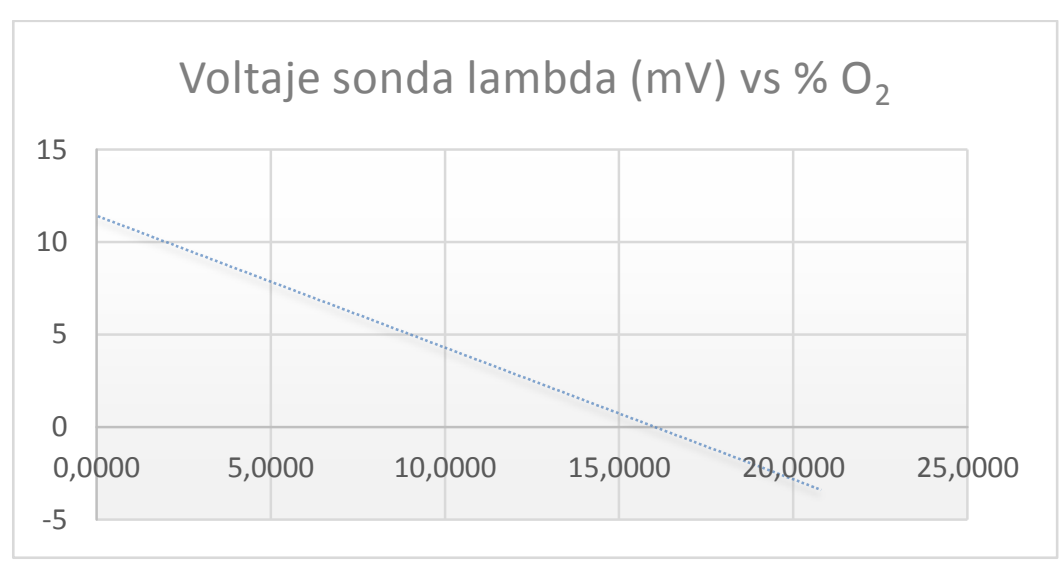

Figura 3: Curva de voltaje medido con la sonda lambda vs $\% \mathrm{O}_{2}$

\subsection{Acondicionamiento de la señal}

La sonda lambda emite una señal de voltaje pequeña y fue necesario acondicionar la señal con una ganancia de 10 veces su valor inicial. Una señal con rangos de voltaje amplios, permite una mayor resolución de medición. El voltaje de salida del amplificador se determina mediante la ecuación 1.

$$
\text { Vout }=\operatorname{Vin}\left(1+\frac{R 2}{R 1}\right)
$$

Donde la ganancia es;

$$
1+\frac{R 2}{R 1} \text { y se representa con la letra } A
$$

El valor de resistencia $R 2$ para una ganancia $A=10$ y un valor de $R 1=1 \mathrm{k} \Omega$ se haya:

$$
\begin{gathered}
10=1+\frac{R 2}{R 1} \\
9=\frac{R 2}{1 k \Omega} \\
9 k \Omega=R 2
\end{gathered}
$$

La señal amplificada 10 veces muestra valores desde los $-340 \mathrm{mV}$ hasta los $1000 \mathrm{mV}$. Estos valores aun no pueden ser leídos por ningún indicador de instrumentación y se debe diseñar un circuito que permita estandarizar o acondicionar la señal y obtener valores que oscilen desde 0 a 
10 voltios. La ecuación de la señal amplificada es naturalmente la de una recta también y se corresponde con la ecuación 2.

$$
y=m x+b
$$

Entonces, el valor de la pendiente de la recta se determina de la forma siguiente:

$$
\begin{gathered}
y-b=m x \\
y-y 1=m(x-x 1)
\end{gathered}
$$

Para acondicionar la señal se debe primeramente graficar una curva de estandarización. Estandarizar una señal significa llevar un valor de voltaje o corriente en un rango amplio a valores estándar que pueden ser, para este caso, un valor de corriente de 4 - 20mA o un voltaje 0 - 10V. En la Figura 4 se muestra la curva de estandarización de una señal de voltaje.

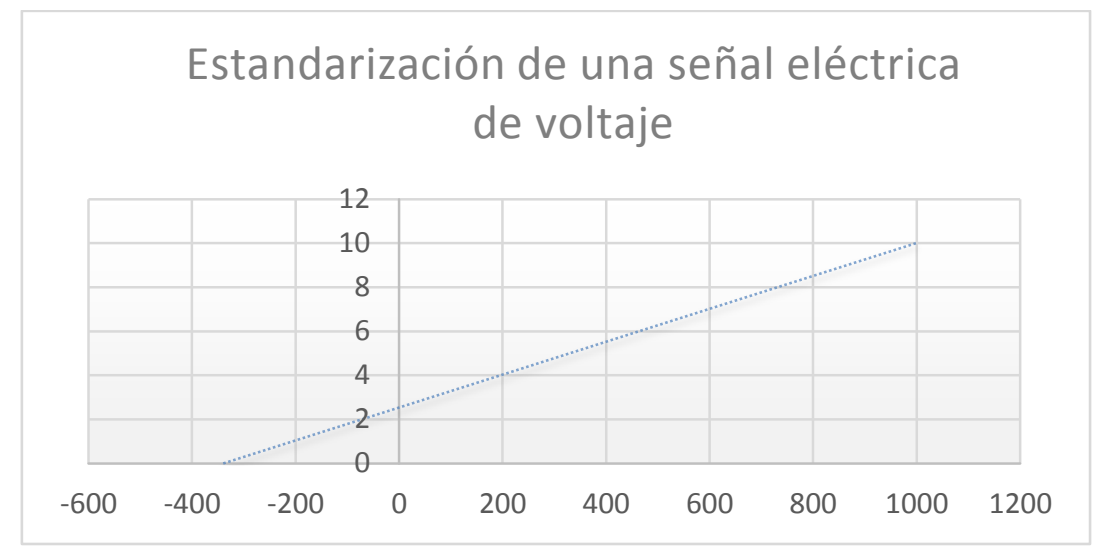

Figura 4: Estandarización de una señal de voltaje

Al desarrollar la ecuación 3 se obtiene la ecuación de estandarización de la señal.

$$
\begin{aligned}
& \frac{y-y 1}{x-x 1}=m \\
& \frac{y-y 1}{x-x 1}=\frac{y 2-y 1}{x 2-x 1}
\end{aligned}
$$

La ecuación 4 es una igualdad de la pendiente de la recta del sensor, a través de ella se puede conocer el valor del voltaje estandarizado en función del voltaje emitido por la sonda lamba. Los valores de $x_{1}$ y $x_{2}$ corresponden a $-340 \mathrm{mV}$ y $1000 \mathrm{mV}$ respectivamente y los valores de $y_{1}$ y $y_{2}$ son los valores estandarizados de $0 \mathrm{~V}$ a $10 \mathrm{~V}$.

$$
\begin{aligned}
& \frac{y-0}{x-(-0.34)}=\frac{10-0}{1-(-0.34)} \\
& y=7.462 x+2.54
\end{aligned}
$$

La ecuación que define el acondicionador de señal se corresponde con la ecuación 5, y está estructurado con un amplificador no inversor de ganancia $A=7,462$ y un sumador que se regula con un potenciómetro para agregar un valor de 2,54 V. El circuito acondicionador completo se muestra en la Figura 5 con sus tres etapas: (Amplificación, Acondicionamiento e Inversión de señal). 


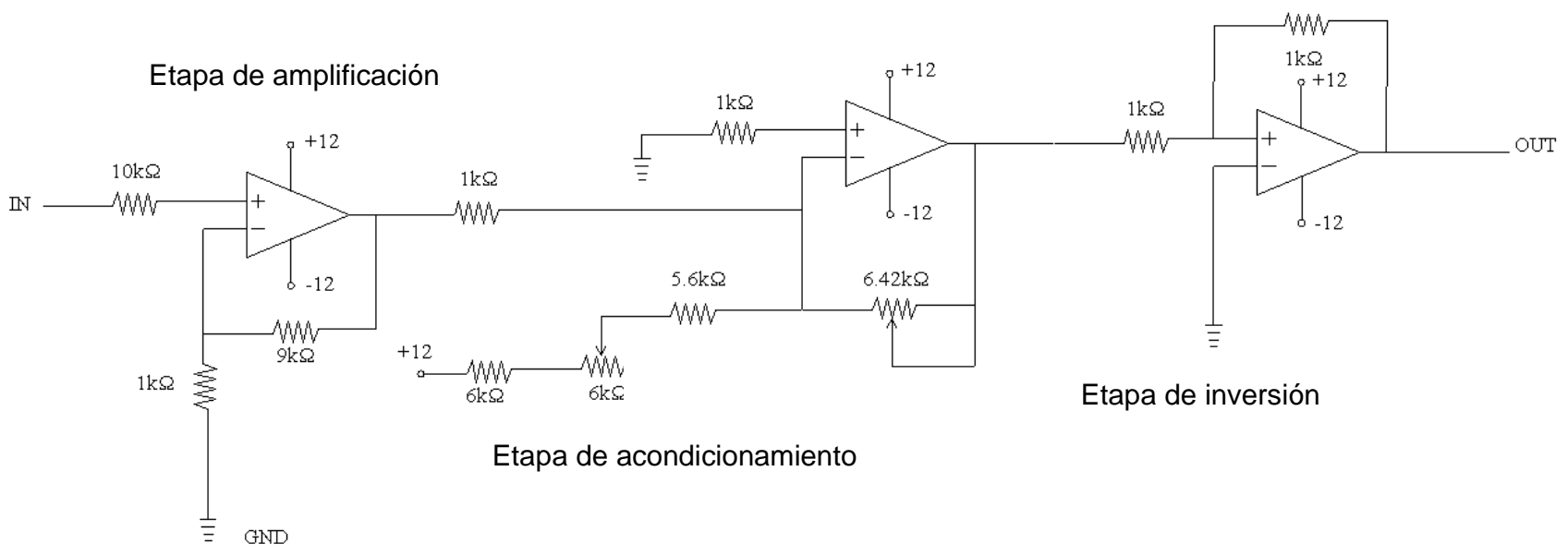

Figura 5: Acondicionador de señal completo

El resultado obtenido con la utilización de amplificadores para el acondicionador de señal, es de signo negativo y para corregirlo y llevarlo a positivo, se utiliza el amplificador inversor de $A=1$.

$$
\begin{gathered}
7.462=1+\frac{R 2}{R 1} \\
6.462=\frac{R 2}{R 1}
\end{gathered}
$$

Se asigna el valor de $1 \mathrm{k} \Omega$ a R1 para determinar el valor de R2;

$$
6.462 \mathrm{k} \Omega=R 2
$$

\subsection{Función lineal en bloques de operación PLC Twido para la obtención de la concentración de oxígeno en porcentaje $\left(\% \mathrm{O}_{2}\right)$.}

Como se observó en la Figura 3 , los valores de voltaje van desde $(\mathrm{y} 1=-3.4 \mathrm{mV}$ hasta y2 $=11.4 \mathrm{mV})$ y el valor de $\mathrm{O}_{2}$ va desde $(x 1=0$ a $\times 2=20.8 \%)$, que es la concentración de oxígeno diatómico. La pendiente de la curva para este caso es decreciente y su valor se determina como se sigue:

$$
\begin{gathered}
m=\frac{y 2-y 1}{x 2-x 1} \\
m=\frac{11.4-(-3.4)}{20.8-0} \\
m=-0.711
\end{gathered}
$$

Como los valores de interés son los del porcentaje de oxígeno, en la ecuación de la recta se despeja la variable $x$. El valor de $b$ es el intersecto en el eje de las ordenadas que es igual a 11.4 v.

$$
x=\frac{y-b}{m}
$$

En la Figura 6 se muestra porcentaje de oxígeno en función del voltaje de la sonda lambda mediante bloques de operación en Twido Suite. 


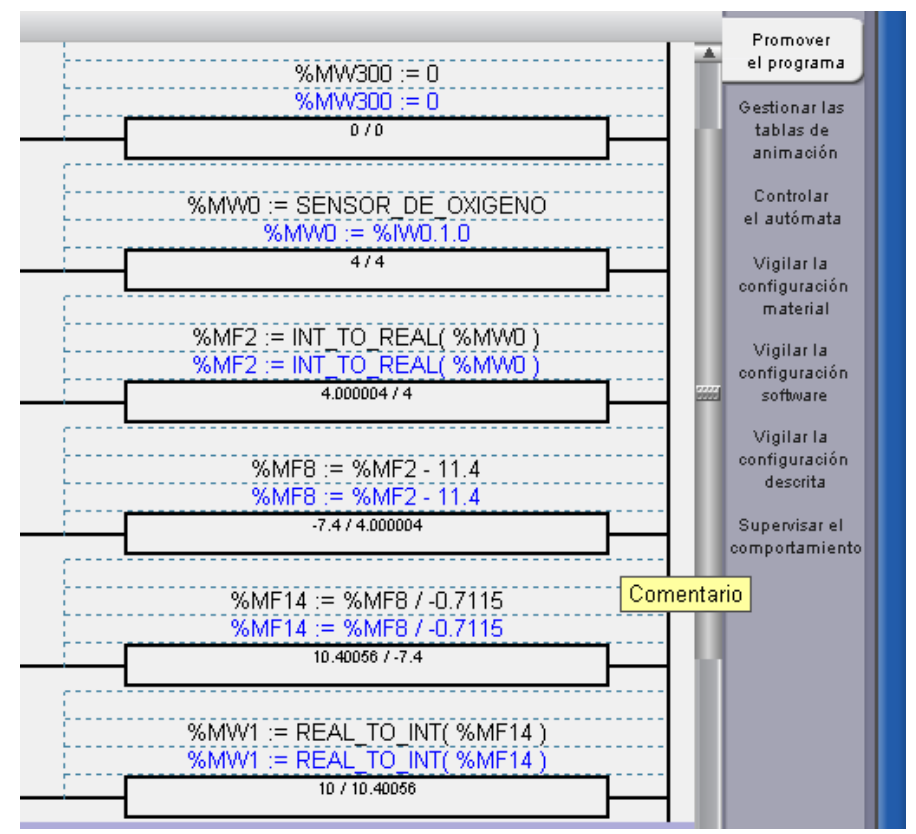

Figura 6: Porcentaje de oxígeno según voltaje de la sonda lambda en bloques de operación en Twido Suite.

Para determinar el valor del $\mathrm{O}_{2}$ e ingresarlo en los bloques de operación del PLC, se resta el valor de voltaje (valores de y) y el intersecto $(b)$, para posteriormente dividirlo entre la pendiente $(m), \mathrm{y}$ se obtiene el valor del porcentaje de oxígeno en función del voltaje de la sonda lambda.

\subsection{Determinación del porcentaje de $\mathrm{CO}_{2}$ a partir del $\mathrm{O}_{2}$}

Cuando se habla de análisis de gases existen variables que son medidas y variables que son calculadas, para este caso el porcentaje de $\mathrm{O}_{2}$ es una variable medida y a partir de este valor y otros parámetros se pueden calcular variables como el porcentaje de $\mathrm{CO}_{2}$, de acuerdo a (Jecht, 2004) la concentración de dióxido de carbono se puede obtener por la expresión:

$$
C O 2=\frac{C O 2 \max x(21-O 2)}{21}
$$

Donde:

$\mathrm{CO}_{2}$ max: Valor específico máximo de $\mathrm{CO}_{2}$ (Fueloil ligero $15,4 \%$ vol. $\mathrm{CO}_{2}$ )

El valor 21: Contenido de oxígeno del aire en (\%)

$\mathrm{O}_{2}$ : Porcentaje de oxígeno medido

En Chinea (2010) se indica la relación existente entre el $\% \mathrm{O}_{2} \mathrm{y} \% \mathrm{CO}_{2}$, la que se muestra en la Tabla 1.

Tabla 1: Relación entre $\% \mathrm{O}_{2}$ y $\% \mathrm{CO}_{2}$

\begin{tabular}{|c|c|c|c|c|c|c|c|c|c|c|c|c|c|c|c|}
\hline$\% \mathrm{CO}_{2}$ & 14.7 & 14.0 & 13.3 & 12.5 & 12.0 & 11.5 & 10.4 & 9.6 & 8.8 & 8.1 & 7.4 & 6.6 & 5.9 & 5.2 & 4.4 \\
\hline$\% \mathrm{O}_{2}$ & 1 & 2 & 3 & 4 & 5 & 6 & 7 & 8 & 9 & 10 & 11 & 12 & 13 & 14 & 15 \\
\hline
\end{tabular}

Fuente: http://www.cubasolar.cu/biblioteca/Ecosolar/Ecosolar30/HTML/articulo06.htm 


\subsection{Implementación de un control proporcional PID}

Para el ingreso de aire se adaptó un servomotor al dámper que controla el ingreso de aire hacia la cámara de combustión, para poder realizar este control se implementó un lazo PID que calcula la diferencia entre la variable real contra la variable deseada como se muestra en la Figura 7.

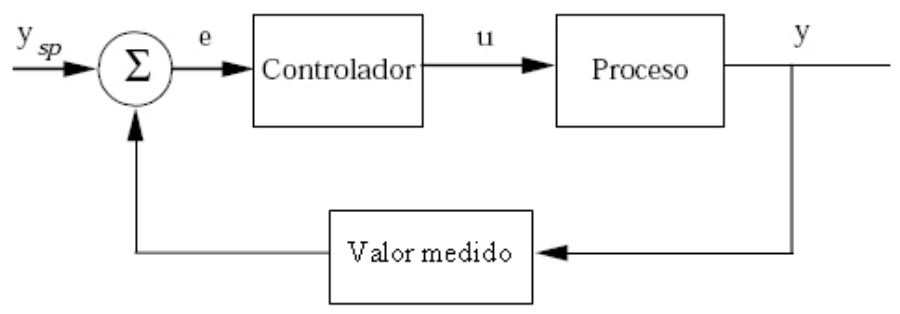

Figura 7: Diagrama de bloques de un proceso con un controlador por realimentación

Fuente: http://www.mailxmail.com/curso-control-pid-avanzado/pid-controladores-realimentacion

En la Figura 7 se muestra un sistema de realimentación mediante un diagrama de bloque. El sistema tiene dos grandes componentes, el proceso y el controlador. El proceso tiene una entrada que es la variable manipulada llamada variable de control y se denota por la letra $u$. La variable de control influye sobre el proceso vía actuador, que suele ser una válvula o motor. La salida del proceso se llama la variable de proceso (PV) y se representa por la letra $y$. Esta variable se mide con un sensor lambda. El actuador y el sensor se consideran parte del bloque etiquetado como "Proceso". El valor deseado de la variable de proceso se llama el punto de consigna (SP) o valor de referencia. Se denota por Ysp. El error de control e es la diferencia entre el punto de consigna y la variable de proceso.

$$
e=Y s p-y
$$

Para la aplicación desarrollada:

$$
\begin{aligned}
& \text { Ysp }=\text { Punto de consigna 4,35 \% de oxígeno (Valor medio del intervalo 3,7 - 5) } \\
& \mathrm{u}=\text { Variable de control ( } 0 \text { - } 5 \mathrm{~V} \text { servomotor) } \\
& \mathrm{y} \quad=\text { Variable de proceso (Porcentaje de oxígeno en gases de combustión) }
\end{aligned}
$$

La implementación de un control PID en el sistema de control, permite la regulación automática del ingreso de aire según el porcentaje de oxígeno detectado. En caso de que la concentración de $\mathrm{O}_{2}$ exceda el valor de $Y s p$, la variable de control, en este caso el servomotor, disminuirá la entrada de aire hasta que el valor medido se estabilice, caso contrario si es baja la contracción de oxígeno en los gases de combustión, el servomotor abrirá el paso de aire hacia la cámara de combustión.

La Figura 8 muestra el submenú de monitoreo de los principales parámetros de la caldera cuando está en funcionamiento. Desde ella se puede acceder directamente a las demás pantallas que forman parte de este sistema de control como son: menú principal, entradas análogas, I/O digitales, Dámper y curvas de comportamiento de diferentes parámetros. 


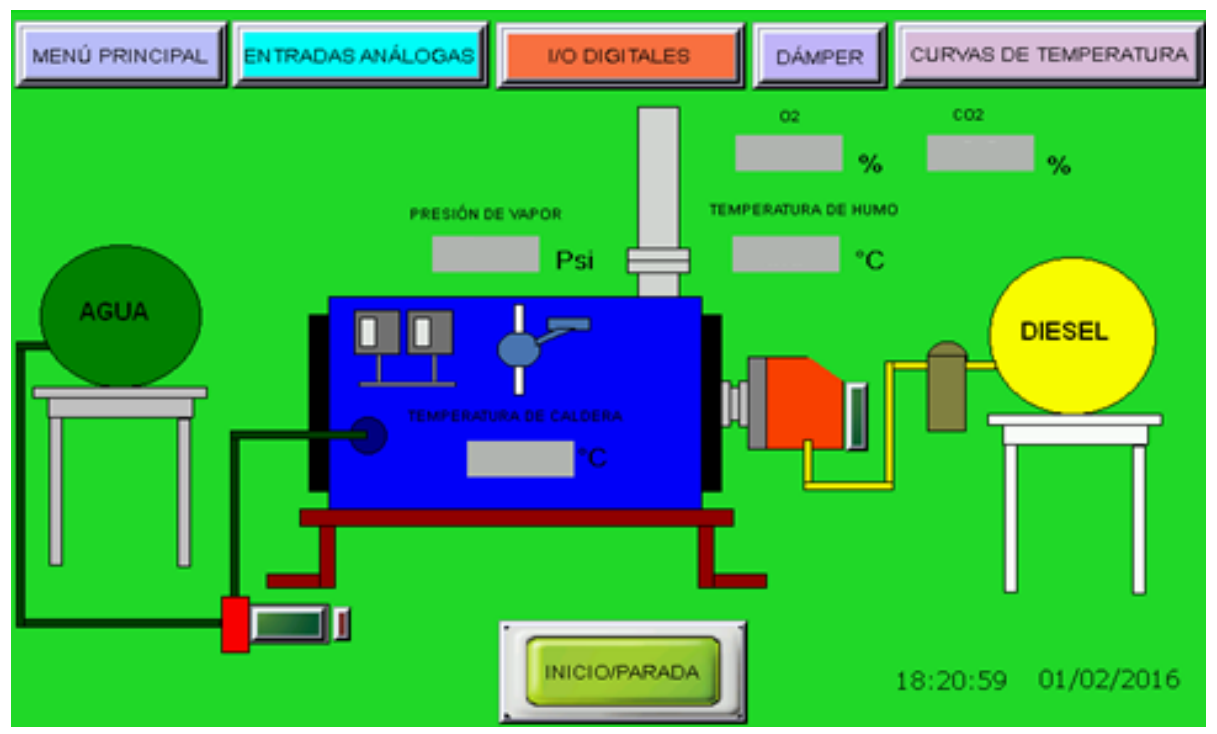

Figura 8: Submenú de Monitoreo

\section{Resultados}

El sistema de control de combustión desarrollado se implementó en una caldera de 5 BHP con diámetro de la chimenea de $10 \mathrm{~cm}$. Los resultados de las lecturas realizadas fueron comparados con los obtenidos al utilizar un analizador de gases marca Testo, modelo 350XL, de la empresa CORPLAB, certificada para estudios de composición de gases de combustión.

Según las normas de Texto Unificado de Legislación Ambiental Secundaria (TULAS), para las calderas con diámetro inferior a $3 \mathrm{~m}$ se deberán utilizar dos puertos de muestreo, ubicados a una distancia de, al menos, ocho diámetros de chimenea corriente abajo y dos diámetros de chimenea corriente arriba de una perturbación en la dirección del flujo normal de gases de combustión, lo que presupone la toma de muestras en un estado de flujo laminar del fluido. Así, se ubicaron los puntos 1 y 2 indicados en la Figura 9. El protocolo del análisis de gases en fuentes fijas aplicado por la empresa CORPLAB establece la toma de tres mediciones en cada punto de interés en condiciones de funcionamiento de la caldera, el valor promedio de las mediciones efectuadas en cada punto se muestra en la Tabla 2. El valor del porciento de $\mathrm{O}_{2}$ en ambos puntos fue semejante ligeramente superior en el punto 2 por esta razón se tomó finalmente el valor de $10.8 \%$ de $\mathrm{O}_{2}$ correspondiente al punto 2 para ser comparado posteriormente con el sistema de control desarrollado.

Tabla 2: Concentración de $\mathrm{O}_{2}$ medido en dos puntos con el analizador de gases marca Testo

\begin{tabular}{|l|c|c|c|c|c|c|}
\hline Punto & Ubicación & $\begin{array}{c}\text { Valor } \\
\text { parcial } \\
\mathbf{1}\end{array}$ & $\begin{array}{c}\text { Valor } \\
\text { parcial } \\
\mathbf{2}\end{array}$ & $\begin{array}{c}\text { Valor } \\
\text { parcial } \\
\mathbf{3}\end{array}$ & $\begin{array}{c}\text { Valor } \\
\text { Promedio } \\
\mathbf{O}_{\mathbf{2}} \text { TESTO }\end{array}$ & observaciones \\
\hline Punto1 & inicio de la chimenea & 10,4 & 10,8 & 10,5 & 10,6 & $\begin{array}{c}\text { valores } \\
\text { normales }\end{array}$ \\
\hline Punto2 & $\begin{array}{c}\text { después de la } \\
\text { perturbación (Codo) }\end{array}$ & 10,6 & 11,1 & 10,7 & 10,8 & $\begin{array}{c}\text { valores } \\
\text { normales }\end{array}$ \\
\hline
\end{tabular}




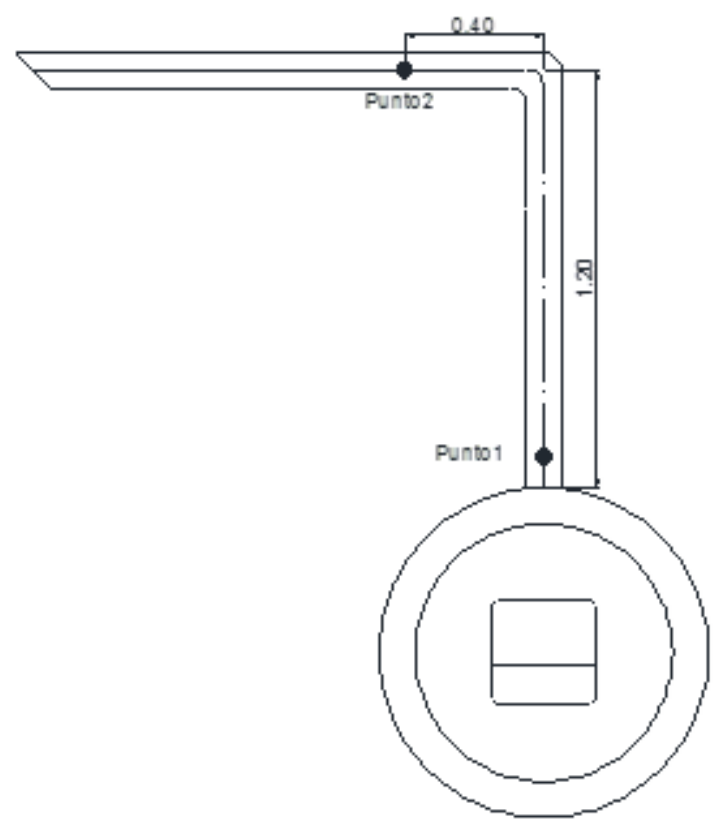

Figura 9: Señalización de los puntos donde fue ubicada la sonda lambda en la chimenea.

Para las mismas condiciones en las que se efectuaron las mediciones con el equipo Testo modelo $350 \mathrm{XL}$, de la empresa CORPLAB en el punto 2 , se realizaron nuevamente tres lecturas en dicho punto ahora con el sistema de control desarrollado determinándose su valor promedio. Este valor se muestra en la Tabla 3 conjuntamente con el valor promedio obtenido al utilizar el equipo Testo.

Tabla 3: Comparación de los valores promedios de la concentración de $\mathrm{O}_{2}$ medidos con el sistema de control implementado y con el equipo Testo modelo 350XL, de la empresa CORPLAB.

\begin{tabular}{|c|c|c|c|c|c|}
\hline Punto & Ubicación & $\begin{array}{c}\text { Valor } \\
\text { medido } \\
\% \mathrm{O}_{2}\end{array}$ & $\begin{array}{c}\text { Valor } \\
\text { medido } \\
\% \mathrm{O}_{2} \\
\text { TESTO }\end{array}$ & $\begin{array}{c}\text { Diferencia } \\
\%\end{array}$ & Observaciones \\
\hline Punto2 & $\begin{array}{c}\text { Después de la } \\
\text { perturbación } \\
\left(\text { Codo } 90^{\circ}\right)\end{array}$ & 10.48 & 10.8 & 0,32 & $\begin{array}{c}\text { Rango tolerable } 1.3 \% \\
\text { (comprendido entre los valores } \\
\left.\text { recomendados de } 3.7 \mathrm{y} 5 \% \text { de } \mathrm{O}_{2}\right)\end{array}$ \\
\hline
\end{tabular}

Posteriormente, con la caldera a pleno funcionamiento, se reguló la graduación del dámper de ingreso de aire hasta comprobar que la caldera trabajara dentro del rango comprendido entre el 3,7 y el $5 \%$ de $\mathrm{O}_{2}$ recomendado.

Al existir una descompensación en la concentración de oxígeno medido por el sistema de control, es decir, que los valores captados se encuentren fuera de los límites establecidos, el sistema mediante el control proporcional PID, compensará el valor de oxígeno faltante o excedente dentro de la cámara de combustión a través de la apertura o cierre del dámper de ingreso de aire según corresponda, asegurándose así que el porcentaje de $\mathrm{O}_{2}$ presente en los gases de combustión se encuentre dentro del rango recomendado. 
Los valores captados en las mediciones efectuadas en el punto 2 para la nueva posición del dámper con el sistema en pleno funcionamiento se muestran en la Tabla 4.

Tabla 4: Valores de $\% \mathrm{O}_{2}$ medidos en el punto 2 de muestreo con el nuevo sistema de control implementado

\begin{tabular}{|c|c|c|c|c|c|c|}
\hline Punto & Ubicación & $\begin{array}{c}\text { Valor } \\
\text { parcial } \\
\mathbf{1}\end{array}$ & $\begin{array}{c}\text { Valor } \\
\text { parcial } \\
\mathbf{2}\end{array}$ & $\begin{array}{c}\text { Valor } \\
\text { parcial } \\
\mathbf{3}\end{array}$ & $\begin{array}{c}\text { Valor } \\
\text { Promedio } \\
\mathbf{O}_{\mathbf{2}}\end{array}$ & observaciones \\
\hline Punto 2 & $\begin{array}{c}\text { Después de la } \\
\text { perturbación } \\
\left(\text { Codo } 90^{\circ}\right)\end{array}$ & 4,6 & 5,1 & 4,7 & 4,8 & $\begin{array}{c}\text { Valores } \\
\text { normales }\end{array}$ \\
\hline
\end{tabular}

\section{Discusión}

Al considerar las lecturas del equipo Testo modelo $350 \mathrm{XL}$ como valor referencial en cada punto de medición. Tal como pudo observarse en la Tabla 3, la diferencia en las lecturas del porcentaje de $\mathrm{O}_{2}$ en el punto 2 medido con la sonda lambda del sistema de control desarrollado respecto a su correspondiente valor referencial, no supera el valor de $0.32 \%$, muy inferior al 1,3\% comprendido entre los valores límites del intervalo de 3,7 y $5 \% \mathrm{O}_{2}$ para el uso del diésel como combustible, lo que demuestra la factibilidad del empleo del sistema de control implementado para la lectura del porcentaje de $\mathrm{O}_{2}$ presente en los gases de combustión de la caldera.

Los valores obtenidos inicialmente en el punto 2 cercanos al $10 \%$ de $\mathrm{O}_{2}$ tanto con el equipo Testo como con el sistema de control implementado (Ver Tabla 2), corroboran la descalibración de la caldera cinco años después de su puesta en funcionamiento. Sin embargo, para poder establecer el paralelo entre las mediciones realizadas por la empresa CORPLAB y las efectuadas con el sistema de control implementado, fue preciso mantener las mismas condiciones de entrada de aire (graduación del dámper) pese a conocerse que los valores obtenidos estaban fuera del rango admisible.

El valor promedio de las mediciones efectuadas en el punto 2 mostrado en la Tabla 4 al utilizar el sistema de control implementado, se encuentra dentro del rango de concentración de $\mathrm{O}_{2}$ recomendado para los gases de combustión del diésel. Lo que asegura la formación de una mezcla estequiométrica con el mejor aprovechamiento posible de la capacidad calorífica del combustible, la reducción del tiempo de operación y del consumo de combustible durante el funcionamiento de la caldera. El control automático de la relación aire/combustible con el sistema de control implementado asegura la calidad de los gases de combustión de la caldera de manera desatendida, con implicación favorable en la protección del medio ambiente y en la mejor utilización de la fuerza de trabajo.

El sistema de control implementado puede aplicarse no solo a fuentes fijas de combustión, puede acoplarse también a otros sistemas que usan la combustión para producir poder calorífico, como el de secado de materias primas. El procedimiento seguido y los resultados alcanzados en la 
presente investigación pueden servir de orientación metodológica para la instalación de sistemas de control de gases de la combustión mediante pantallas HMI y PLC en procesos análogos.

\section{Conclusiones y Recomendaciones}

Se realizó el diseño e implementación de un control de combustión de una caldera pirotubular de 5 BHP a diésel, mediante el cual se mejora la calidad de la mezcla airecombustible, al mantener el porcentaje de $\mathrm{O}_{2}$ presente en los gases de combustión dentro del límite establecido para este combustible.

Como elementos principales del control de combustión fueron utilizados, una pantalla HMI de marca DELTA, touch screen con despliegue gráfico, un PLC modular Twido TWLMDA20DRT el que estará encargado de accionar sobre el actuador para el control de la entrada de oxígeno en función del valor de la lectura realizada por la sonda lambda.

Fue necesario acondicionar la señal de voltaje de $-34 \mathrm{mV}$ a $100 \mathrm{mV}$ emitida por la sonda lambda, con una ganancia de igual a 10, para luego estandarizar dicha señal y obtener valores de voltaje en el intervalo de 0 a 10 voltios con la finalidad de lograr compatibilizar dicha señal con el voltaje admitido por la entrada analógica del PLC utilizado.

La función que relaciona los valores de voltaje entregados por la sonda lambda con el porcentaje de oxígeno suministrado por el PLC se corresponde con la ecuación de una recta, la que permite obtener el valor de porcentaje de oxígeno presente en los gases de combustión de manera continua durante el funcionamiento de la caldera.

Se obtuvo una diferencia en la captación del porciento de oxígeno en los gases de combustión de la caldera al utilizar el sistema de control implementado de solo $0.32 \%$, respecto al valor obtenido en las mediciones realizadas por la empresa certificada CORPLAB, con el equipo Testo modelo 350XL. Este valor es inferior al valor de $1.3 \%$ comprendido en el intervalo de 3,7 a $5 \%$ de $\mathrm{O}_{2}$, recomendado para gases de la combustión del diésel, lo que demuestra la factibilidad del empleo de la sonda lambda en la lectura de la contaminación de gases de combustión de dicha caldera.

Finalmente, como recomendación del trabajo se tiene que la operación y mantenimiento del sistema de control de gases de la combustión que ha sido implementado deberá ser realizada por personas capacitadas para evitar daños en el sistema de control de este equipo conforme al nivel de la tecnología utilizada. 


\section{Bibliografía}

Álvarez, Saúl. (2006). Generadores de vapor. Programa de Ingeniería Biomédica, Universidad Nacional Experimental "Francisco de Miranda", Venezuela.

Chinea, Dr. Jesús M. Guzmán. 2010. Cubasolar. Cubasolar. [En línea] 20 de Mayo de 2010. http://www.cubasolar.cu/biblioteca/Ecosolar/Ecosolar30/HTML/articulo06.htm.

Jecht, Dr. Ulrich. (2004). Análisis de gases de combustión en la industria. Madrid.

Kojan, Anthony L. (2000). Manual de calderas. Madrid: Mc Graw-Hill, ISBN: 139788448125462.

Linares, C. y Díaz, J. (2015). Las PM 2,5 y su afección a la salud. Recuperado 12 Nov. 2015 de: https://www.um.es/estructura/servicios/sprevencion/cseguridad/documentos/EE_58_PM2 5.pdf

Ministerio del Ambiente (2007). Norma de emisiones al aire desde fuentes fijas de combustión. Quito: Publicaciones del Ministerio del Ambiente.

Ministerio del Ambiente (2012). Texto Unificado de Legislación Ambiental Secundaria (TULAS)

Salinas Villar, Antonio. (2006). Motores. Madrid, España: Thomson, 2006. 